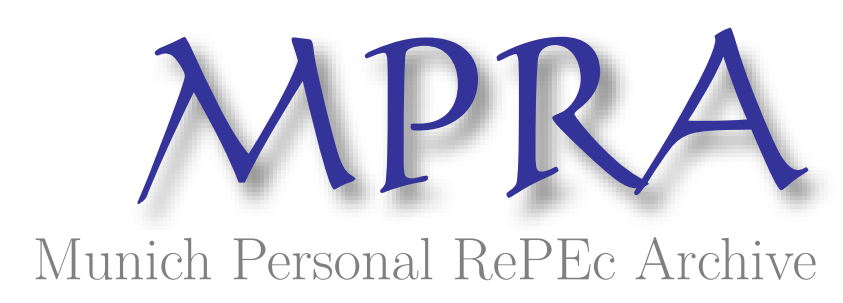

\title{
The Role of Liquidity Individuals in the Decision-Making
}

Steinbacher, Matjaz

2009

Online at https://mpra.ub.uni-muenchen.de/13566/

MPRA Paper No. 13566, posted 23 Feb 2009 05:23 UTC 


\title{
THE ROLE OF LIQUIDITY INDIVIDUALS IN THE DECISION-MAKING
}

\author{
Matjaz Steinbacher \\ Steinbacher.si \\ Financial analyst and advisor \\ Prvomajska ulica 7 \\ SI - 2310 Slovenska Bistrica, Slovenia \\ e-mail: matjaz.steinbacher@gmail.si \\ Phone: +38641960928
}

\begin{abstract}
We simulate social network games of a portfolio selection to analyze the role of liquidity individuals for the developments in individuals' decision-making in financial markets. Liquidity individuals prove to be a significant element in the decision-making process of the entire network, as they keep the information of non-dominant strategies alive. Their role is especially significant under omniscient individuals, whereas a little less under non-omniscient individuals. As long as individuals do not lose the information of all the alternatives, their role is insignificant.
\end{abstract}

Keywords: liquidity individuals, social networks, decision-making, portfolio analysis, stochastic finance.

JEL Classification: G11, C73. 


\section{Introduction}

Decisions of individuals on financial markets can generally be described through uncertainty, seeking for information about the securities, and social networks (Axelrod 1984; Watts and Strogatz 1998; Wasserman and Faust 1994), with preferences, knowledge and other individual specific characteristics its key elements. Individuals on the markets are non-omniscient individuals each possessing a small bit of the entire knowledge (Hayek 1945). To say that individuals acquire information they need from their presence in the social networks is to say that individuals do not only learn from their experience, but also from the experiences of others. Such processes induce herd behavior and lead to synchronous decisions in time (Bikhchandani et al. 1998; Banerjee 1992).

In the model, we assume that individuals change the strategy they use and pick the one that they believe will bring them highest outcome. This is done by observing past outcomes of the strategies they use, or past outcomes of the efficiencies of the strategies used by individuals to whom they are connected. However, we also assume that some of individuals never change the alternative they choose in the beginning, no matter of its efficiency or the efficiency of the alternatives. This is in line with Rubinstein (1998) and Osborne and Rubinstein (1990) and findings from the behavioral studies (Hirshleifer 2001). As individuals face a tradeoff between the complexity of one strategy on one side and its efficiency and prospects of a successful implementation of the strategy on the other they are much less prone to changing the strategy they satisfactorily pursue for some time. This has several different effects to the outcome of the game. Not only this corresponds to the preferences of individuals, but such individuals also appear as liquidity individuals in the game, keeping the information about the non-dominant alternatives alive and available to the others in the network throughout the game. Without such individuals, some consecutive worse outcomes of some alternatives might convey that such alternatives cease to exist. Despite they might be worthwhile in the longer run. In this article, we consider that deficiency. 
Games are simulated using real data, with individuals using social networks and choosing between the stocks of two bank corporations, Citigroup (NYSE: C) and CreditSuisse (NYSE: CS), or the portfolio of the two. Section 2 presents the model in short and Section 3 the results. Article ends with short concluding remarks.

\section{General framework}

\subsection{The model}

There are $A=(1,2, \ldots, 1000)$ infinitely lived agents, distributed on the lattice and connected in the small world network (Watts and Strogatz 1998; Wasserman and Faust 1994). On average, each individual in the network is connected to six others.

The network $g=(V, E)$ is a set of vertices $V=\left\{v_{1}, v_{2}, \ldots, v_{1000}\right\}$, representing agents, and edges $E=\left\{e_{1}, e_{2}, \ldots, e_{n}\right\}$, representing their pairwise relations. If two agents are connected, we denote $i j \in g$, while $i j \notin g$ represents two unconnected vertices. Using adjacency matrix, $i j=1$ if $i j \in g$ and $i j=0$ if $i j \notin g$. We use undirected graph, where edges are unordered pairs of vertices, thus if $i j=1 \Leftrightarrow j i=1$. In a small world network, people have many local and some global connections with others, which we get by rewiring some of the connections.

According to their initial preferences, individuals are split into two groups; the first consists of those who prefer Citigroup stocks, and the second of those who prefer CreditSuisse stocks. The share of individuals who prefer stocks of CreditSuisse are denoted $0 \leq u \leq 1$, whereas $(1-u)$ individuals prefer Citigroup stocks. Individuals from both groups can choose between pure strategy, either to opt for Citigroup or CreditSuisse stocks or make a portfolio of the two. We denote with $C$, if individuals who prefer Citigroup stocks decide for pure strategies and 
$C p$ if they opt for a portfolio. Individuals preferring CrediSuisse stocks and decide for pure CreditSuisse stocks strategies are denoted $C S$ and $C S p$ if they opt for a portfolio. In either case, portfolio is selected from the part of stocks one prefers, $0 \leq p i \leq 1$, while remainder $(1-p i)$ represents stocks of the other company. Individuals accumulate their wealth in time according to the strategy they choose, while they can change between the strategies in every period during the game.

$$
\begin{aligned}
& W_{t+1}\left(A_{C}\right)=W_{t}\left(A_{C}\right) \cdot[1+C r] \\
& W_{t+1}\left(A_{C p}\right)=W_{t}\left(A_{C p}\right) \cdot[1+C r \cdot p i+C S r \cdot(1-p i)] \\
& W_{t+1}\left(A_{C S p}\right)=W_{t}\left(A_{C S p}\right) \cdot[1+C S r \cdot p i+C r \cdot(1-p i)] \\
& W_{t+1}\left(A_{C S}\right)=W_{t}\left(A_{C S}\right) \cdot[1+C S r]
\end{aligned}
$$

$W_{t+1}(\bullet)$ and $W_{t}(\bullet)$ represent wealth of an individual in time $t$ and $t+1$, while (•) denotes the strategy played by an individual in time. Returns of stocks, denoted $\mathrm{Cr}$ and $\mathrm{CSr}$, are exogenous to the individuals and they cannot foresee them, neither do they know the system how prices change in time. There is a discrete time interval on $t=(1,2, \ldots, 2457)$. The level of omniscience of agents is defined through Fermi logistic probability function (Szabó and Töke 1998) as

$$
\wp=\left[1+\exp \left[\left(W\left(A_{i}\right)-W\left(A_{j}\right)\right) / \kappa\right]\right]^{-1}
$$

This means that in every time period $t$ an agent $A_{i}$ chooses one of the individuals to which he is directly connected, $A_{j}$, and compares his payoff, $W\left(A_{i}\right)$, to the payoff of a selected individual, $W\left(A_{j}\right)$. It depends upon the level of coefficient $\kappa$ which strategy an individual $A_{i}$ will adopt. For $\kappa=0$, individual always adopts the strategy that gives higher outcome. Such individual is said to be omniscient. Higher the value of $\kappa$ and smaller the difference between the two payoffs, more likely it is that an individual does not choose the strategy with 
higher payoff. Such individual is characterized as non-omniscient. Contrary, lower the value of a coefficient $\kappa$ and bigger the difference between the two payoffs, more likely it is that an individual chooses the strategy with higher payoff. In simulations, we use omniscient individual with $\kappa=0.001$ and nonomniscient with $\kappa=1.0$.

We assume that a portion of individuals, denoted $l \in(0,1)$, never change their strategies. Such individuals assure the liquidity of all alternatives in the game and could be named liquidity individuals. We set $l=0.1$ and assume that whatever their strategy, these are individuals numbered from $901 \leq n \leq 1000$ from $n=1000$ individuals. In the games, liquidity individuals pursue all four alternatives, which are randomly defined in the beginning of the games.

\subsection{Data}

Data is used from finance.yahoo.com portal. Data refer to adjusted closed prices of both stocks from 21.1.1999 until 19.11.2008. An adjusted closed price is a price adjusted for splits and dividends. In order to use the same time-period for both stocks, we omit adjusted closed prices for the stock for a time units if the other stock was inactive on that day.

\section{Simulation results}

\subsection{Omniscient individuals}

We first simulate individual games with omniscient individuals under different initial values of $u$ and plot their entire developments.

$$
u=0.5
$$


We first choose $u=0.5$ and $p i=0.3$, whereas $u$ is changing in time and $p i$ is constant. We make two independent realizations of the game and plot the results in Figures $1 \mathrm{a}$ and $1 \mathrm{~b} . C$ in figures represent the share of individuals that choose Citigroup, $C p$ represent the share of individuals who prefer Citigroup stocks and choose for a portfolio. Similarly, $C S$ and $C S p$ represent the shares of individuals who prefer CreditSuisse stocks and opt for pure strategies or portfolio.

Figure 1a: Omniscient individuals pursuing each alternative

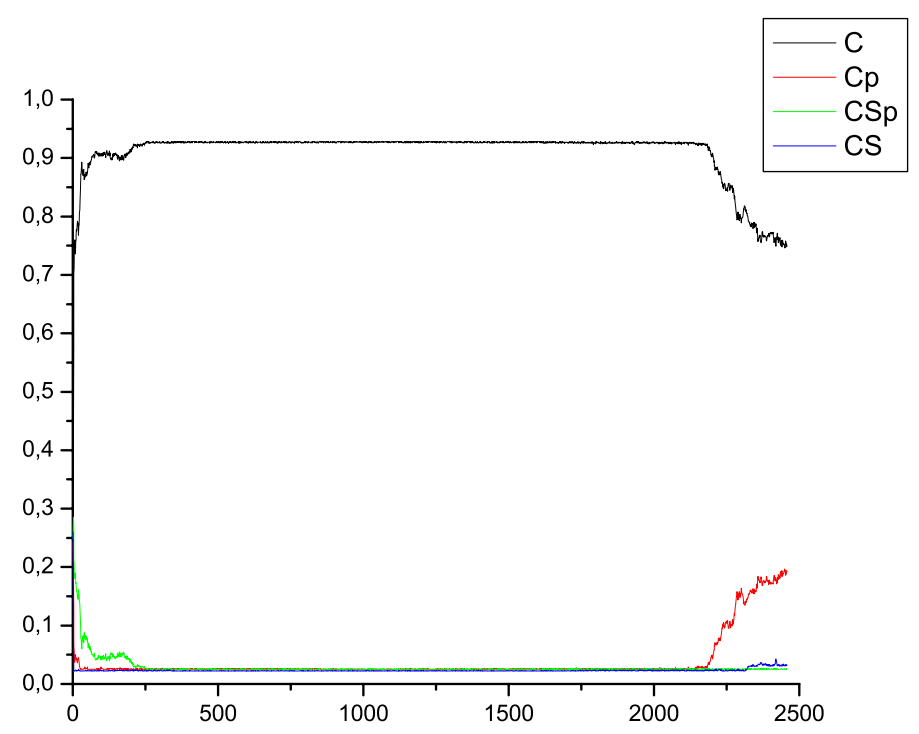

Figure 1b: Omniscient individuals pursuing each alternative 


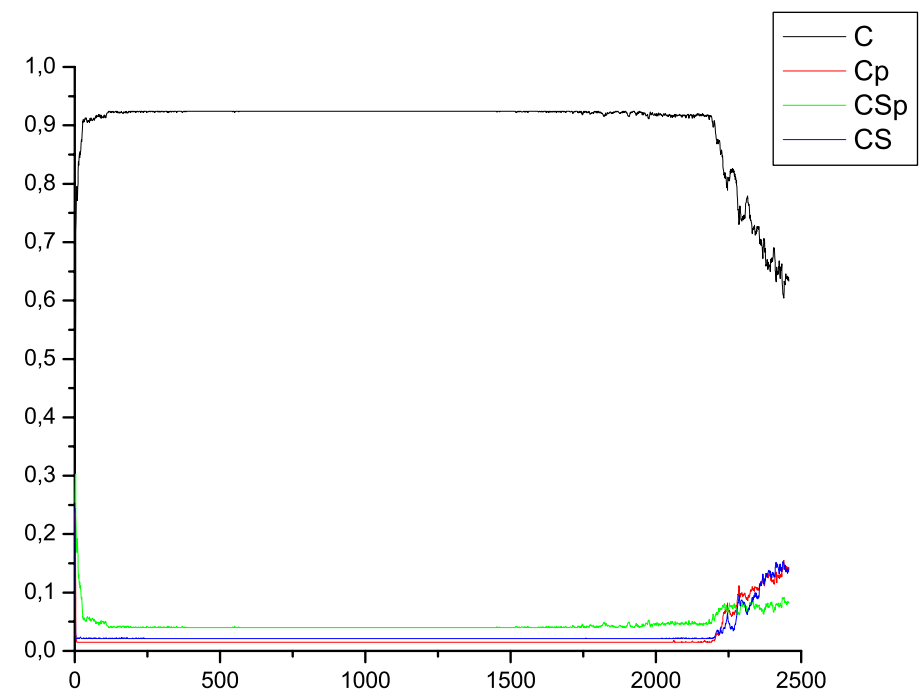

Through great part of the game, the possession of Citigroup stocks is a dominant strategy played by omniscient individuals. Figures clearly demonstrate the significance of liquidity individuals for the developments in the games. As they assure that the information of dominated alternatives are not lost and remain at the disposal to others, individuals are able to switch from once dominant to once dominated strategies as the game proceeds and circumstances in the game change.

In the games, we see that the presence of liquidity individuals leads to the decline of the share of individuals possessing only stocks of Citigroup and towards the alternatives that include CreditSuisse stocks. With no liquidity individuals, such switch is not possible as individuals lose the information about the alternative strategies, which happens in the early stages of the game.

$$
u=0.8
$$

We now increase the initial share of individuals preferring stocks of CreditSuisse, thus $u=0.8$. The value of $p i=0.3$ is constant in time. The shares of individuals opting for each alternative are plotted in Figure 2a for the first realization of the game and in Figure $2 b$ for the second.

Figure 2a: Omniscient individuals pursuing each alternative 


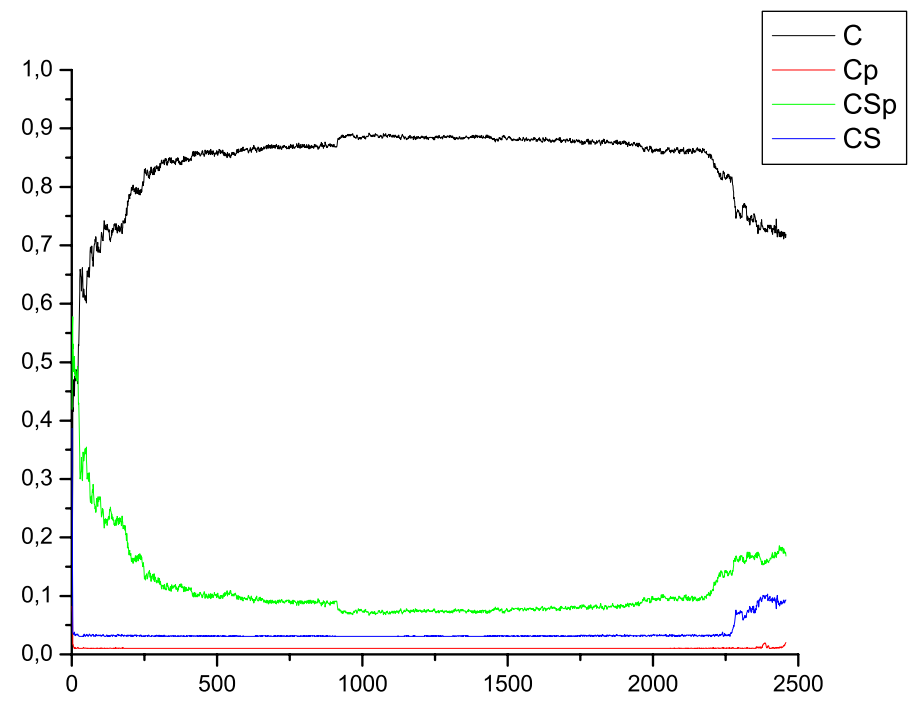

Figure 2b: Omniscient individuals pursuing each alternative

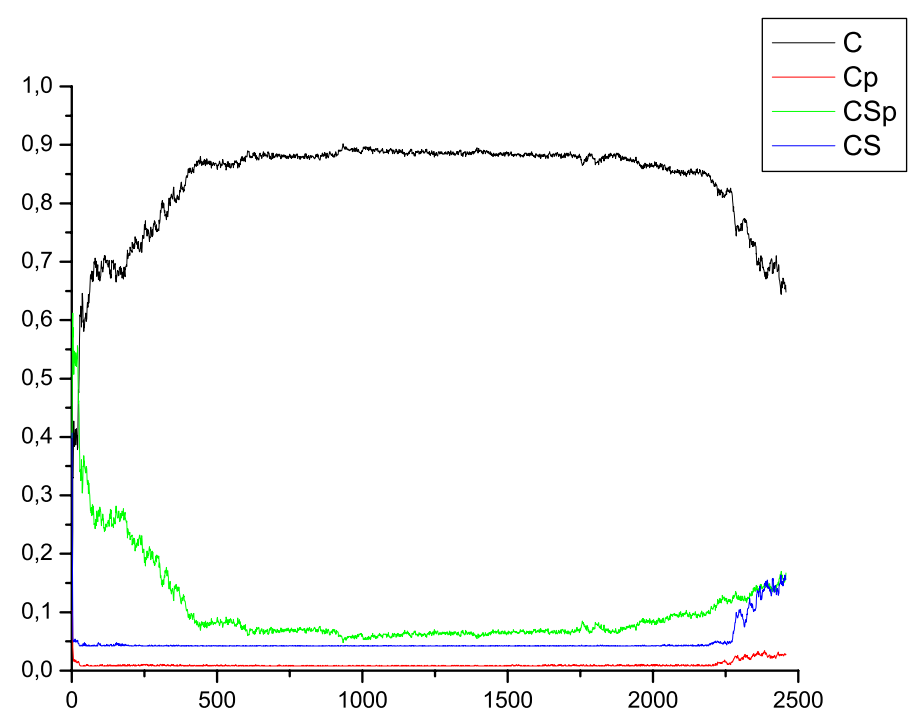

Again, we see that the role of liquidity individuals for the developments of the games is significant, as they keep the information of all the alternatives alive throughout the game, for which they all could be used by all the rest as the game proceeds. 


\subsection{Non-omniscient individuals}

We now turn to non-omniscient agents with $\kappa=1.0$ and make simulations of individual games under different values of variables $u$ and $p i$.

$$
u=0.5
$$

Again, groups of individuals preferring each stock are in the start of equal size, thus $u=0.5$, and are changing in time, while $p i=0.3$ is constant throughout the game. Figure $3 \mathrm{a}$ depicts the shares of individuals choosing each alternative in the first realization of the game and Figure $3 \mathrm{~b}$ present the results of the second realization of the game.

Figure 3a: Non-omniscient individuals pursuing each alternative

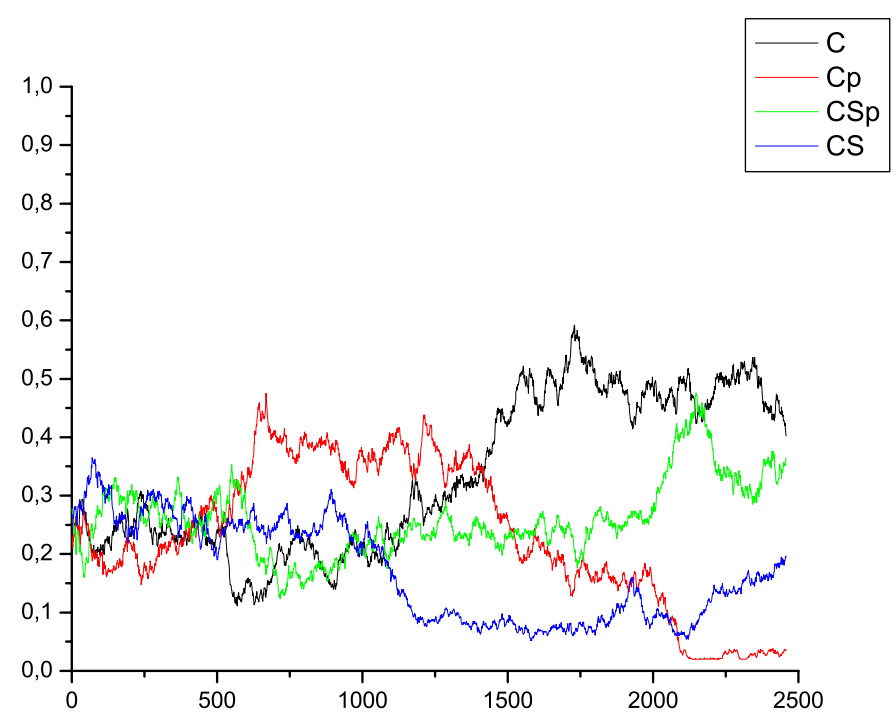

Figure 3b: Non-omniscient individuals pursuing each alternative 


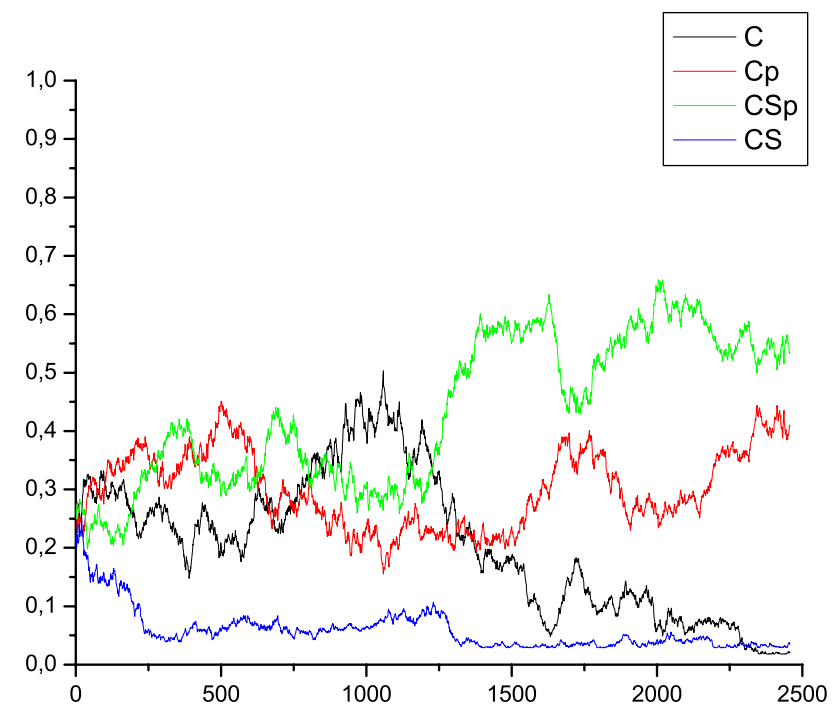

From the Figures, we see that the role of non-omniscient individuals is not as significant for the liquidity of alternative strategies as is the role of omniscient individuals. This is because non-omniscient individuals do not react so prompt to the changing circumstances as omniscient individuals do, for which they do not lose the information about the alternative investments as fast as omniscient individuals do. However, in Figure 3a liquidity individuals are significant for the existence of the alternative $C p$ and in Figure $3 b$ for alternatives $C S$ and $C$.

$$
u=0.8
$$

Again, groups of individuals preferring each stock are in the start of equal size, thus $u=0.8$, and are changing in time, while $p i=0.3$ is constant throughout the game. Figure $4 \mathrm{a}$ and Figure $4 \mathrm{~b}$ present the results of the two independent realizations of the games.

Figure 4a: Non-omniscient individuals pursuing each alternative 


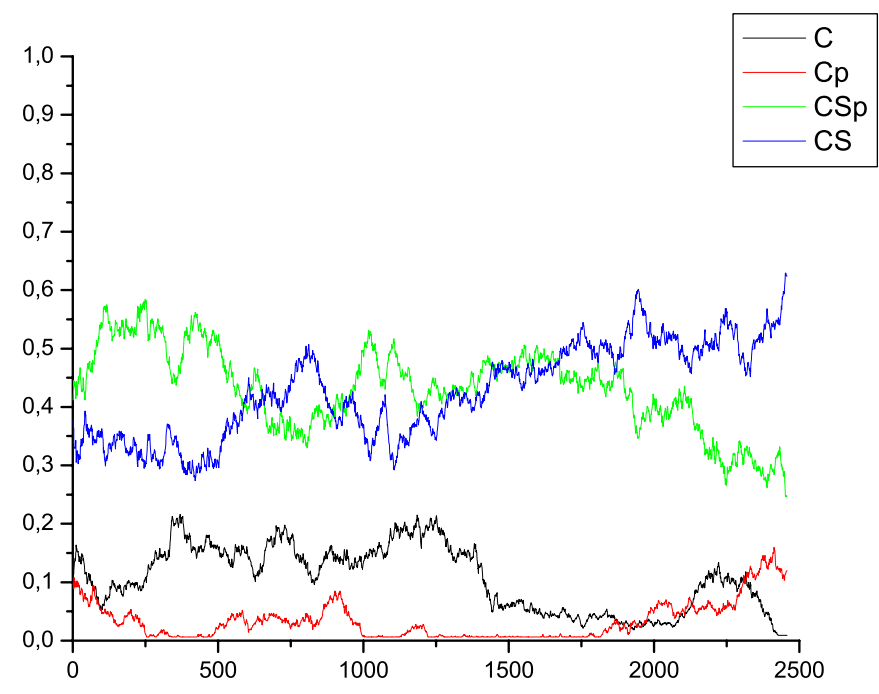

Figure 4b: Non-omniscient individuals pursuing each alternative

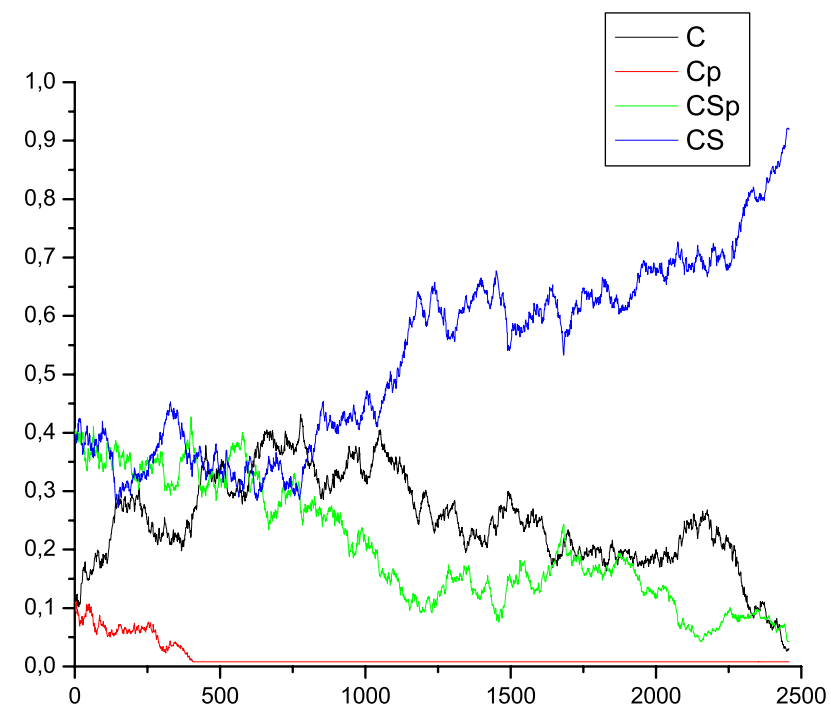

The role of liquidity individuals is nicely represented in the Figure 4a and Figure $4 \mathrm{~b}$. In both realizations of the game, liquidity individuals prove to be significant for the existence of the alternative $C p$. In the first realization of the game $C p$ alternative was used by others three times after liquidity individuals kept it alive (Figure 4a), whereas in the second realization only liquidity individuals pursue a $C p$ alternative from the time interval $t=406$ on (Figure $4 \mathrm{~b}$ ). In time intervals close to the end of the game, liquidity individuals prove to be a decisive element 
in keeping the information of the alternative $C$ alive, which is necessary for the liquidity of a stock. This shift reflects the effects of the financial crisis, which put stocks of Citigroup much lower than the stocks of CreditSuisse.

\section{Concluding remarks}

In the article, we feature the role liquidity individuals have for the developments of the decision-making in the portfolio management. We demonstrate the decisiveness of the role of such liquidity individuals in the process of the decision-making of all the individuals in the network, working according to the principle of invisible hand. Because they keep the information of the all nondominant alternatives alive, they prevent such information to get lost, which would happen with no such individuals in the network. Therefore, all alternatives, despite once strictly dominated, remain liquid and ready to be pursued as the game proceeds and the relevant information of the alternatives change.

\section{REFERENCES}

1. Axelrod, R. (1984). The Evolution of Cooperation. New York: Basic Books.

2. Banerjee, A. (1992). A Simple Model of Herd Behavior. Quarterly Journal of Economics, 107(3), 797-818.

3. Bikhchandani, S., Hirshleifer, D., Welch I. (1998). Learning from the Behavior of Others: Conformity, Fads, and Information Cascades. Journal of Economic Perspectives, 12(3), 151-170.

4. Hayek, F.A. (1945). The Use of Knowledge in Society. American Economic Review, 35(4), 519-530.

5. Hirshleifer, D. (2001). Investor Psychology and Asset Pricing. Journal of Finance, 56(4), 1533-1597.

6. Osborne, M., Rubinstein, A. (1990). Bargaining and Markets. New York: Academic Press.

7. Rubinstein, A. (1998). Modeling Bounded Rationality. Cambridge: MIT Press.

8. Szabó, G., Töke C. (1998). Evolutionary Prisoner's Dilemma Game on a Square Lattice. Physical Review E, 58(1), 69-73.

9. Wasserman, S., Faust K. (1998). Social Network Analysis: Methods and Applications. Cambridge: Cambridge University Press.

10. Watts, D., Strogatz S. (1998). Collective Dynamics of Small World Networks. Nature, 393(4), 440-442. 
$C$, plotted with black line, represents the share of agents who prefer Citigroup stocks and play pure strategies, $C p$, plotted with red line, represents the share of agents who prefer Citigroup stocks and opt for a portfolio. $C S$, plotted with green line, and CSp, plotted with blue line, represent shares of agents who prefer CreditSuisse stocks and opt for pure strategies or portfolio. 